\title{
Abdominal extranodal lymphoma detection: positron emission tomography/ computed tomography can help
}

\author{
Mohsen Ahmed Abdelmohsen ${ }^{1 *}$ (D) and Walid Mohamed Omar²
}

\begin{abstract}
Background: Lymphoid neoplasms that present with masses are broadly referred to as lymphomas, and they can be classified to two main groups: Hodgkin's $(\mathrm{HL})$ and non-Hodgkin's lymphoma (NHL); they are mainly characterized by enlargement of lymph nodes (nodal disease) although any organ in the body can be involved in different settings of the disease (extranodal disease). Adequate staging, which has a direct impact on prognosis, is essential to properly plan therapy. Stage IV disease with extranodal dissemination should be treated by long-term systemic chemotherapy. By adding the metabolic changes to the conventional $C T$ morphologic changes, combined positron emission tomography (PET) and computed tomography (CT) may offer clinically useful addition in staging of lymphomas and detection of extranodal lesions.
\end{abstract}

Results: The study included thirty nine patients, with forty-eight extranodal sites with lymphomatous infiltration, twenty-four males (61.5\%), and fifteen females (38.4\%). The patients showed forty-eight extranodal abdominal lymphomatous infiltration. The study included twelve gastric lymphomatous lesions (25\%), twelve splenic lymphomatous lesion (25\%), three ileo-caecal lymphomatous lesions (6.25\%), three patients (6.25\%) with skin infiltration, three adrenal lymphomatous lesions (6.25\%), three patients with hepatic lymphoma, and twelve bone marrow infiltrations (25\%).

The most accurate SUV max cutoff value among studied cases was 3.5, the highest SUV max value noted was 21 in gastric lesions, and the lowest SUV max noted was 4.1 in adrenal lesions.

Confirmation of PET/CT results was done by histopathological assessment and post-management follow-up.

Conclusion: PET/CT study is an effective tool for evaluation of extranodal lymphomas. PET/CT fusion images can affect the clinical management plan by detection of extranodal lymphomas with lymphoma staging modification.

Keywords: Extranodal lymphoma, Positron emission tomography/computed tomography, Standardized uptake value

\section{Background}

There are multiple classification systems of lymphomas, the main histopathological classification of lymphomas into Hodgkin's disease (40\%) and non-Hodgkin's lymphomas $(60 \%)$ and several further classifications according to the WHO classification systems in 2008 with about 43 subtypes of lymphomas divided into 4 main groups: mature B cell

\footnotetext{
* Correspondence: Mohsenmohsenmd@yahoo.com

'Department of Radio-diagnosis and Intervention, Faculty of Medicine, University of Alexandria, 10 Shamplion street, Elazareeta, Alexandria, Egypt Full list of author information is available at the end of the article
}

lymhpoma, mature T cell lymphoma, Hodgkin's disease, and post-transplant disorders $[1,2]$.

Extranodal lymphomas, particularly in cases of NHL, can involve in any organ. While secondary extension from a disseminated form is the most frequent forming stage IV disseminated lymphomas, isolated extranodal primary lesions, although rare, are possible forming stage IE lymphomas. The criteria for such a primary lesion are based on the absence of distant sites (lymph nodes outside of the adjacent drainage area, spleen, bone marrow, or any other distant lymphoid structure) and on the absence of circulating lymphomatous cells, and 
thus conflict with any possible involvement by contiguity or with a stage IV disseminated lymphoma [3].

Whatever organ is concerned, these lymphoma lesions will have a number of common imaging characteristics which are bound to suggest this diagnosis; unfortunately, there are multiple imaging morphological patterns that can mimic other diseases and make the conventional imaging techniques not enough for diagnosis, staging, and assessment of response following chemotherapy or radiotherapy $[3,4]$.

By adding the functional metabolic data to the morphological data obtained in the fused PET/CT images, advance in extranodal lymphomas knowledge can be achieved including its detection, characterization, staging, and assessment of treatment response $[5,6]$.

\section{Methods}

This is a prospective analysis of thirty nine patients with forty-eight pathologically proven abdominal organs extranodal lymphomatous infiltration.

We classified the extranodal lesions to two groups:

Group I: known extranodal lymphomatous infiltration proved by histopathology and PET/CT was done for staging searching for lymphoma elsewhere (thirty-three extranodal sites).

Group II: known patient with history of nodal

lymphoma with detection of extranodal sites on PET/

CT study (fifteen extranodal sites).

The visual assessment method and semi-quantitative analysis were used with measuring the standard uptake value (SUV) max, and the normal would be the normal blood pool liver SUV max.

\section{Reference standard}

All the patients' lesions were subjected to a biopsy when accessible.

\section{Technique}

A hybrid PET/CT scanner (Siemens Biograph 64 PET/ CT scanner) was used in this study .Before the examination by $6 \mathrm{~h}$, the patients were instructed to fast, except for water. Avoidance of (extreme) exercise for at least 6 $\mathrm{h}$ before the study was asked to minimize FDG uptake in muscles and to reduce the false positive results. The allowable blood glucose level for the study was of $<150$ $\mathrm{mg} / \mathrm{dL}$. Voiding of urine was done before injection, and then approximately $5 \mathrm{MBq} / \mathrm{kg}$ body weight of FDG was injected. Sixty to ninety minutes later, the data were acquired [7]. The patients then were kept lying comfortably and were asked not to talk to avoid false positive uptake. A non-contrast-enhanced CT from mid-thigh to the skull was obtained $60 \mathrm{~min}$ after the tracer injection.
Table 1 Anatomical distribution of the extras nodal lymphomatous lesions (nine patients showing two extranodal lymphomatous locations)

\begin{tabular}{lll}
\hline $\begin{array}{l}\text { Anatomical location of the extranodal } \\
\text { lymphomatous infiltration }\end{array}$ & $\begin{array}{l}\text { Number } \\
(\boldsymbol{N}, 48)\end{array}$ & Percentage \\
\hline Splenic & 12 & $25 \%$ \\
Gastric & 12 & $25 \%$ \\
Hepatic & 3 & $6.25 \%$ \\
Skin & 3 & $6.25 \%$ \\
Ossoeus & 12 & $25 \%$ \\
Ileo-caecal & 3 & $6.25 \%$ \\
Adrenal & 3 & $6.25 \%$ \\
Total & 48 & $100 \%$ \\
\hline
\end{tabular}

Nuclear medicine consultant and radiology consultant interpreted the data at the Siemens work station.

\section{Staging system}

Lugano classification.

\section{Statistical analysis}

Qualitative data were described using numbers and percent.

\section{Results}

The study included thirty-nine patients, twenty-four males (61.54\%), and fifteen females (38.46\%) with forty-eight extranodal sites of lymphomatous infiltration including twelve gastric lymphomatous lesions (25\%), twelve splenic lymphomatous lesion (25\%), three ileo-caecal lymphomatous lesions $(6.25 \%)$, three patients $(6.25 \%)$ with skin infiltration, three adrenal lymphomatous lesions (6.25\%), three patients with hepatic lymphoma, and twelve bone marrow infiltrations (25\%) (Table 1).

The study included different pathological types of FDG-avid lymphoma, of the thirty-nine cases: fifteen cases with mucosa-associated lymphoid tissue lymphoma (MALT), twelve cases with diffuse large B cell lymphoma, three cases with anaplastic large $\mathrm{T}$ cell lymphoma, and nine cases with Hodgkin's disease (HD) (Table 2).

The SUV max cutoff value among studied cases was 3.5, the highest SUV max value was 21 noted in gastric

Table 2 Distribution of the patients according to the histopathological types of extranodal lymphoma

\begin{tabular}{lll}
\hline $\begin{array}{l}\text { Histo-pathological type of } \\
\text { lymphomatous infiltration }\end{array}$ & Number $(\boldsymbol{N}, 39)$ & Percentage \\
\hline MALT & 15 & $38.46 \%$ \\
Hodgkin's disease & 9 & $23.07 \%$ \\
Large B cell lymphoma & 12 & $30.70 \%$ \\
Anaplastic large T cell lymphoma & 3 & $7.61 \%$ \\
Total & 39 & $100 \%$ \\
\hline
\end{tabular}



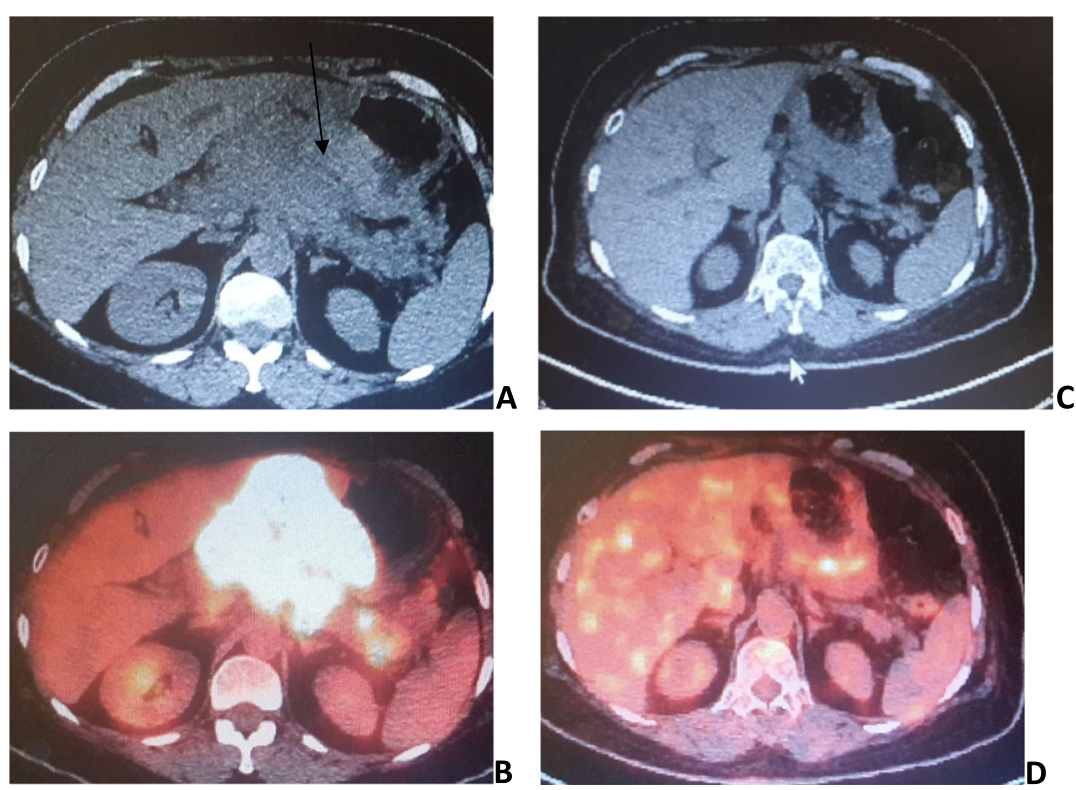

Fig. 1 A 49-year-old female patient with a history of enoscopically proven gastric MALT lymphoma (a) and axial CT abdomen (b) corresponding PET/CT-fused image showing gastric circumferential mural thickening forming a mass merging with gastrohepatic ligament-based soft tissue density (black arrow in a) with avid uptake in the PET/CT fused image by the visual assessment method. c Axial CT abdomen (d) corresponding PET/CT-fused image 5 months later showing regressive course following treatment with residual gastric mural thickening.
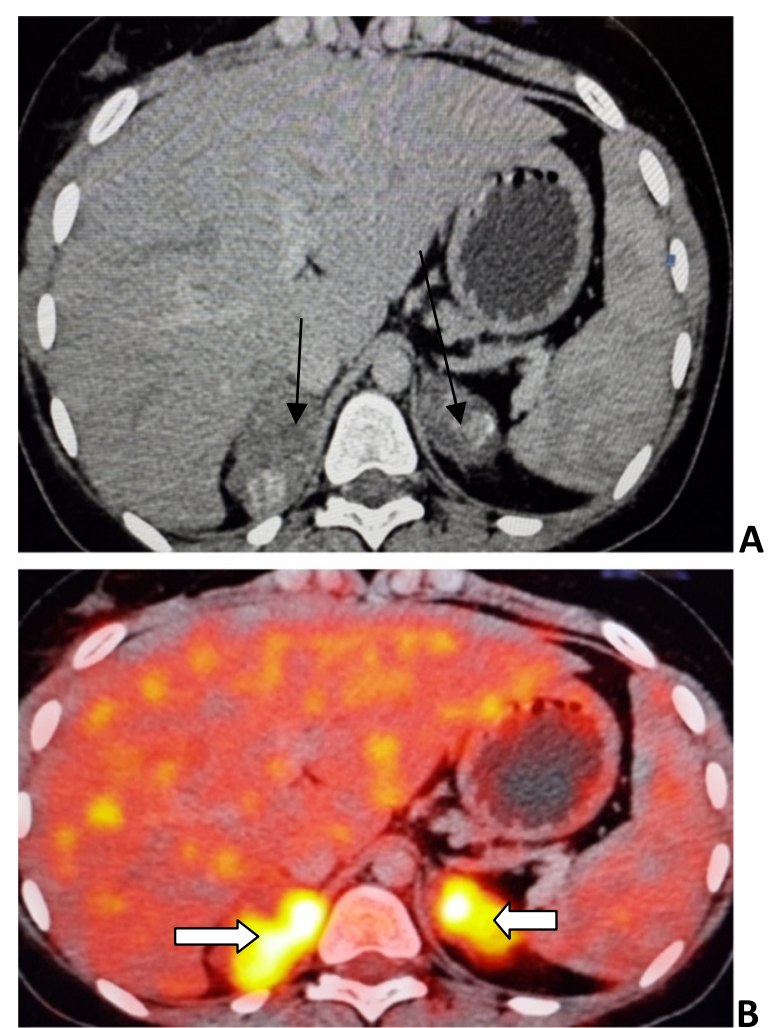

Fig. 2 A 40-year-old female patient with history of NHL presented with bilateral adrenal masses. a Contrast-enhanced CT study showing bilateral hypodense adrenal masses with areas of calcification (black arrows). b PET/CT fused image with bilateral avid FDG uptake by the visual assessment method (open white arrows) lymphoma (Fig. 1), the lowest SUV max value was 4.1 noted in adrenal lymphoma (Fig. 2), and the median SUV value was 9 .

Confirmation of PET/CT results was done by histopathological assessment.

The scored interval inter-observer \% of agreement was $33.3 \%$.

PET/CT modified initial CT staging in twenty-six patients (66.7\%), by using Lugano Classification system, and staging of extranodal lymphomas was distributed between stage IE (single extranodal site) (9 patients) and stage IV (disseminated disease) (17 patients).

Staging was not modified in thirteen patients (33\%). Three patients of them with sole splenic lymphoma and abdominal lymphadenopathy where the splenic involvement considered nodal according to Lugano staging did not alter staging of lymphoma (Table 3) (Fig. 3).

Table 3 Effect of fused PET/CT study in modification of the staging of lymphoma

\begin{tabular}{lll}
\hline & Number $(\boldsymbol{N}, 39)$ & Percentage \\
\hline Modified staging & $\mathbf{2 6}$ & $\mathbf{6 6 . 7 \%}$ \\
- To stage IE & 9 & $34.4 \%$ \\
- To stage IV & 17 & $65.6 \%$ \\
Non-modified staging & $\mathbf{1 3}$ & $\mathbf{3 3 . 3} \%$ \\
-Stage IE & 10 & $76.9 \%$ \\
-Stage I & 3 & $23.09 \%$ \\
Total & 39 & $100 \%$ \\
\hline
\end{tabular}




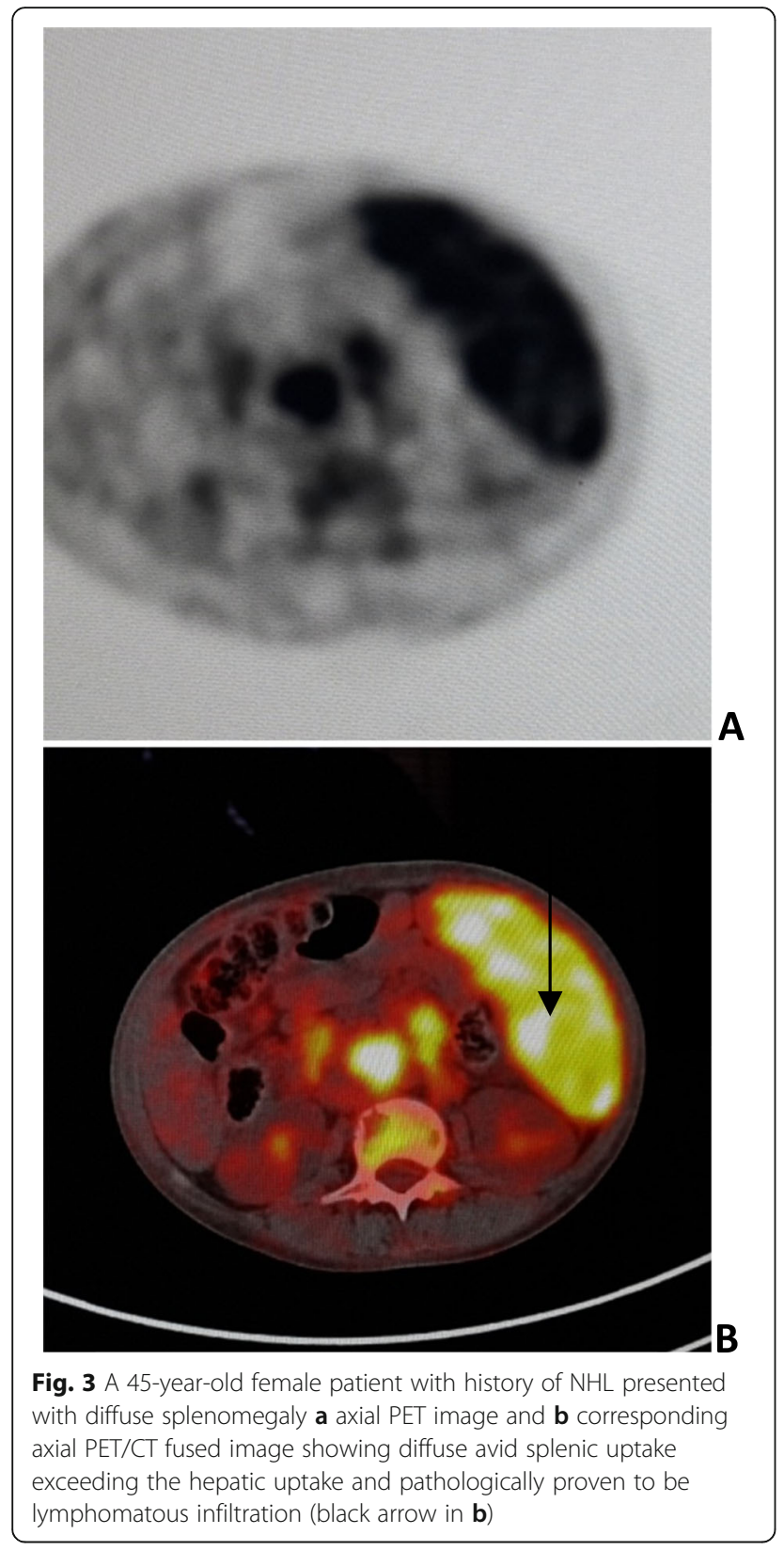

\section{Discussion}

Management of lymphoma depends on accurate staging of lymphoma, while stages I and II are considered as limited disease with local field radiation therapy the 1st line of management, and stages III and IV are considered advanced disease with systemic chemotherapy regimen plus or minus radiotherapy which is the management of choice [8].

PET/CT study offers a chance for complete assessment for extranodal lymphomatous infiltration and allows proper staging of lymphoma; recent studies indicate that $\mathrm{PET} / \mathrm{CT}$ is superior to CT in detecting extranodal disease in the abdomen, especially in the spleen and liver [9].
In this study, Lugano classification was used for staging considering the splenic involvement as nodal disease; among twelve patients with splenic involvement in this study, PET/CT modified staging in nine patients (9/ 12): six patients with splenic and ossoeus involvement (Fig. 4) and three patients with splenic and hepatic involvement, while in three patients (3/12) with nodal and splenic involvement with no effect on the initial CT staging of lymphoma [10], many other studies considered the spleen as extranodal organ, and other studies
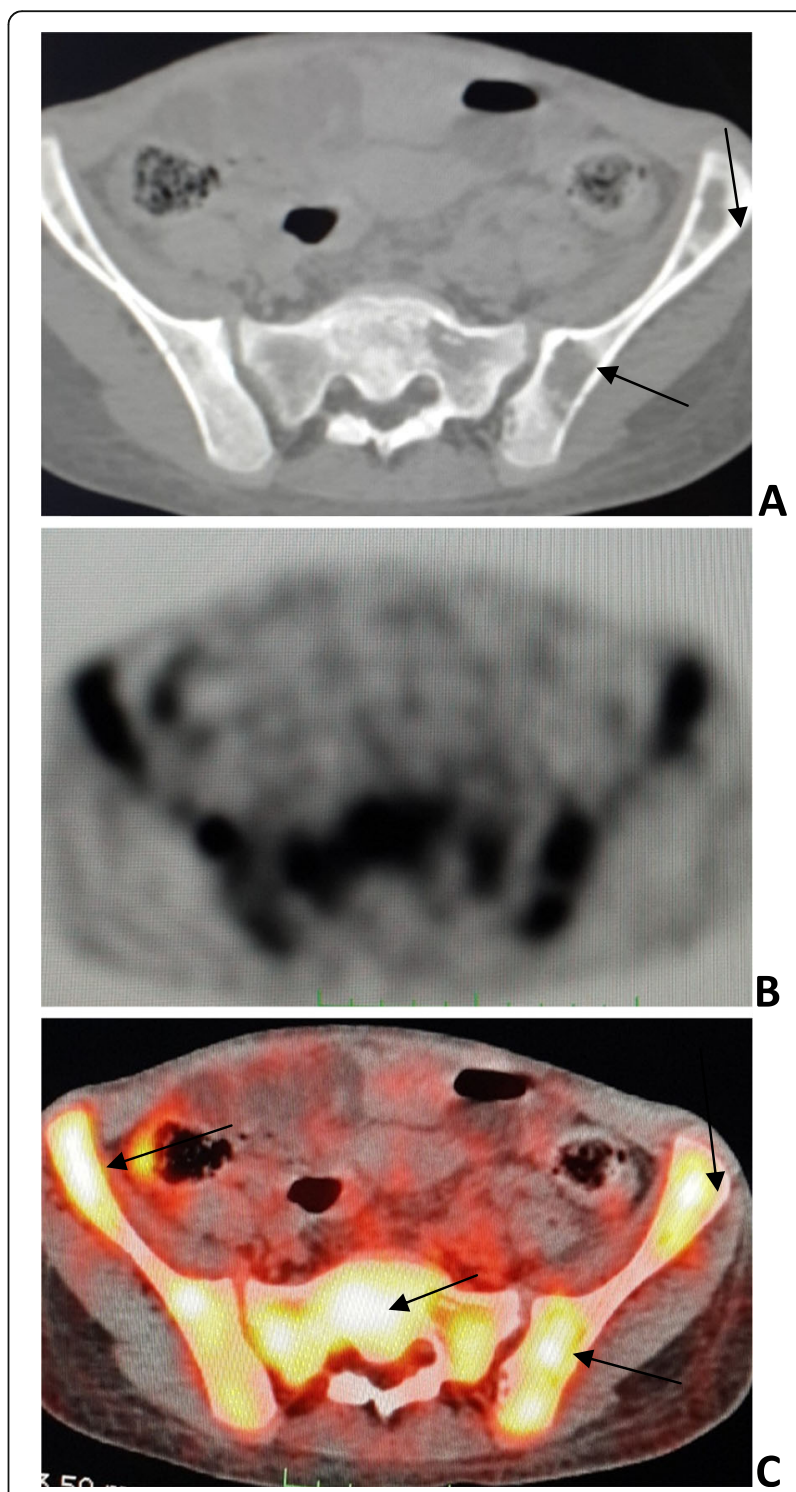

Fig. 4 A 55-year-old male patient with cervical nodal lesions proved to be NHL now presented with relative altered ossoeus density of the pelvic bones notably the left iliac bone with ill-defined lytic medullary areas (arrows in a). b Corresponding axial PET and PET/CT (c) fused image showing diffuse FDG uptake notably at the anatomical site of the left iliac bone lytic areas (arrows in c) 
considered the spleen as nodal organ in Hodgkin's disease and extranodal organ in non-Hodgkin's lymphoma [9-11].

Hepatic lymphoma manifested in our study by hepatomegaly with multiple nodules represented $6.25 \%$ of our study; Manzella et al. [12] stated different patterns of hepatic lymphoma including single focal lesion, multiple focal lesions, hepatomegaly, and miliary pattern constituting $15 \%$ of all extranodal abdominal lymphomas [12].

In agreement with Manzella et al. and Lee at al.'s [13] study, gastric lymphoma and small bowel lymphoma most common presentation was diffuse infiltration with diffuse mural thickening, and other forms of gastric and bowel infiltration included polypoidal mass, diffuse or focal infiltration, ulcerative lesion, or mucosal nodularity [12-14].

The SUV max cutoff value among studied cases was 3.5 , the highest SUV max value was 21 (noted in gastric lymphoma), the lowest SUV max value was 4.1 (noted in Adrenal lymphoma), and the median SUV value was 9 . Cronin et al. [15] on the other hand depended mainly in his study upon the visual assessment with taking hepatic FDG uptake as the reference standard.

Considerations about cost-effectiveness have not been part of this study but will be necessary, as they will play an increasing role in the near future as the clinical utility of PET/CT will lead to a change in the diagnostic and management strategy of cancer patients [16-18].

\section{Conclusion}

$\mathrm{PET} / \mathrm{CT}$ is essential for detection, accurate staging, and planning of management of lymphoproliferative neoplastic disorders.

\section{Abbreviations \\ PET: Positron emission tomography; CT: Computerized tomography; SUV: Standardized uptake value}

\section{Acknowledgements \\ None}

\section{Authors' contributions}

MA reported the cases. WO reported the cases and helped to draft the manuscript. All authors read and approved the final manuscript.

\section{Funding}

This study had no funding from any resource.

\section{Availability of data and materials}

The data sets used and/or analyzed during the current study are available from the corresponding author on reasonable request.

\section{Ethics approval and consent to participate}

A written informed consent was obtained from all patients included in this study to publish this study data. Approval for this study was obtained from the Research Ethics Committee of Alexandria Faculty of Medicine, (IRB 0001209, FWA 00018699). All study procedures were carried out in accordance with the Declaration of Helsinki regarding research involving human subjects.

\section{Consent for publication}

All the patients included in this study gave written informed consent to publish the data contained within this study.

\section{Competing interests}

There are no conflicts of interests. The authors declare that they have no competing interests.

\section{Author details}

${ }^{1}$ Department of Radio-diagnosis and Intervention, Faculty of Medicine, University of Alexandria, 10 Shamplion street, Elazareeta, Alexandria, Egypt. ${ }^{2}$ Department of Nuclear Medicine and Molecular Imaging, National Cancer Institute, Cairo University, Giza, Egypt.

Received: 17 December 2019 Accepted: 26 June 2020

Published online: 09 July 2020

\section{References}

1. Juweid ME, Stroobants S, Hoekstra OS et al (2007) Imaging Subcommittee of International Harmonization Project in lymphoma. Use of positron emission tomography for response assessment of lymphoma: consensus of the imaging Subcommittee of International Harmonization Project in lymphoma. J Clin Oncol 25:571-578

2. Medeiros L, Spagnolo D (2020) Lymphoma 2020: an update on recent advances in pathobiology and application to clinical practice. Pathology 52(1):1-5

3. Paes F, Kalkanis D, Sideras P, Serafini A (2010) FDG PET/CT of extranodal involvement in non-Hodgkin lymphoma and Hodgkin disease. Radiographics 30:269-291

4. Wirth A, Foo M, Seymour JF, Macmanus MP, Hicks RJ (2008) Impact of [18f] fluorodeoxyglucose positron emission tomography on staging and management of early-stage follicular non-Hodgkin lymphoma. Int J Radiat Oncol Biol Phys 71:213-219

5. Metser U, Goor O, Lerman H, Naparstek E, Even-Sapir E (2004) PET-CT of extranodal lymphoma. AJR 182:1579-1586

6. Othman A, Nasr M, Abdel-Kawi M (2019) Beyond lymph nodes: 18F-FDG PET/CT in detection of unusual sites of extranodal lymphoma. Egypt J Radiol Nucl Med 50(1):29

7. Zidan D, Hasan M, Tantawy M (2013) Postoperative restaging: PET/CT impact on diagnosis and management. The Egyptian Journal of Radiology and Nuclear Medicine 44(2):321-329

8. Freudenberg LS, Antoch $G$, Schütt $P$ et al (2004) FDG-PET/CT in re-staging of patients with lymphoma. Eur J Nucl Med Mol Imaging 31:325-329

9. De Jong P A, Van Ufford H MQ , Baarslag et al HJ (2009) CT and18F-FDG PET for noninvasive detection of splenic involvement in patients with malignant lymphoma. Am J Roentgenol 192(3): 745-753

10. Van Heertum R, Scarimbolo R, Wolodzko J, Klencke B, Messmann R et al (2017) Lugano 2014 criteria for assessing FDG-PET/CT in lymphoma: an operational approach for clinical trials. Drug Design, Development and Therapy 11:1719-1728

11. Santos TS, Tavares RS, Farias DL (2017) Splenic marginal zone lymphoma: a literature review of diagnostic and therapeutic challenges. Rev Bras Hematol Hemoter 39:146-154

12. Manzella A, Borba-Filho P, D'Ippolito G, Farias M (2013) Abdominal manifestations of lymphoma: spectrum of imaging features. ISRN Radiology $1: 1-11$

13. Lee W, Lau E, Duddalwar V, Stanley A, Ho Y (2008) Abdominal manifestations of extranodal lymphoma: spectrum of imaging findings. Am J Roentgenol 191(1):198-206

14. Tsang RW, Gospodarowicz MK, Pintilie M et al (2001) Stage I and II MALT Iymphoma: results of treatment with radiotherapy. Int J Radiat Oncol Biol Phys 50:1258-1264

15. Cronin C, Swords R, Truong M, Viswanathan C, Rohren E, Giles F et al (2010) Clinical utility of PET/CT in lymphoma. Am J Roentgenol 194(1):W91-W103

16. Siegel RL, Miller KD, Jemal A (2017) Cancer statistics. CA Cancer J Clin 67:7-30

17. Han A, Xue J, Zhu D, Zheng J, Yue J, Yu J (2011) Clinical value of (18)F-FDG $\mathrm{PET} / \mathrm{CT}$ in postoperative monitoring for patients with colorectal carcinoma. Cancer Epidemiol 35:497-500

18. Cheson B (2018) PET/CT in lymphoma: current overview and future directions. Semin Nucl Med 48(1):76-81

\section{Publisher's Note}

Springer Nature remains neutral with regard to jurisdictional claims in published maps and institutional affiliations. 